\title{
Social inequalities in early childhood health and development: a European-wide systematic review
}

\author{
Demetris Pillas ${ }^{1,2}$, Michael Marmot ${ }^{1}$, Kiyuri Naicker ${ }^{3}$, Peter Goldblatt' ${ }^{1}$, Joana Morrison ${ }^{1}$ and Hynek Pikhart ${ }^{1}$
}

The evidence examining the relationship between specific social factors and early childhood health and developmental outcomes has never been systematically collated or synthesized. This review aims to identify the key social factors operating at the household, neighborhood, and country levels that drive inequalities in child health and development. Medline and CHICOS (a European child-cohort inventory) were systematically searched to identify all European studies published within the past 10 y. 13,270 Medline articles and 77 European child cohorts were searched, identifying 201 studies from 32 European countries. Neighborhood deprivation, lower parental income/wealth, educational attainment, and occupational social class, higher parental job strain, parental unemployment, lack of housing tenure, and household material deprivation were identified as the key social factors associated with a wide range of adverse child health and developmental outcomes. Similar association trends were observed across most European countries, with only minor country-level differences. Multiple adverse social factors operating at both the household and neighborhood levels are independently associated with a range of adverse health and developmental outcomes throughout early childhood. The social gradient in health and developmental outcomes observed throughout the remaining life course may be partly explained by gradients initiated in early childhood.

$\mathbf{T}$ he period of early childhood, defined as the period between prenatal development to $8 \mathrm{y}$ of age, is increasingly recognized as the most crucial period of life span development (1) and the most highly sensitive to external influences (2). During this period, the foundations are laid for every individual's physical and mental capacities, influencing their subsequent growth, health, and development throughout the life course. In certain aspects of child health and development, the potential adverse effects of social and biological influences, such as suboptimal infant brain growth, are likely to be irreversible (3). Hence, intervening to improve early childhood health and developmental outcomes is increasingly being suggested as a priority, as potential interventions are expected to have a stronger impact on an individual's life course health and development while also achieving higher returns than later interventions $(4,5)$.

In recognition of the importance of early childhood, the Commission on Social Determinants of Health's Closing the Gap in a Generation report suggests that "equity from the start" should be an essential component of any attempt to improve health outcomes overall and, in particular, address health inequalities (6). The recent WHO EUROPE's Review of Social Determinants and the Health Divide reinforces this message, suggesting that "the strongest instruments to break the vicious circles of disadvantage lie at the start of life" (7). Not surprisingly, two of the most established theories explaining early childhood development posit that all social and biological factors relating to the family/ household (representing the child's microsystem), the neighborhood (mesosystem), and the broader sociopolitical environment (macrosystem) interact in shaping children's health and development $(8,9)$. Programs that aim to improve the social and economical environments in which children grow up, live, and learn may therefore be instrumental in achieving reductions in health and developmental inequalities which span the entire life course. A comprehensive knowledge base detailing which social and economic factors have the most profound influences on early childhood outcomes is therefore a fundamental first step in structuring such interventions. Although a large number of European-based studies exist which examine the relationship between specific social factors operating at the different levels and health and developmental outcomes in early childhood, no study to date has attempted to systematically collate and synthesize the overall evidence provided from this considerable, but widely dispersed, evidence base.

The purpose of this study was to provide a systematic review and synthesis of all the relevant evidence linking social inequality to child health and developmental outcomes in the European region. Such a review will enable the identification of the important social factors operating at the household, neighborhood, and country levels, as well as the evaluation of any important between-country differences. Identifying the wide range of proximal and distal social factors which are likely to be driving inequalities in early childhood will facilitate more evidence-based, clinically relevant, and cost-effective interventional initiatives in the future. 


\section{Inequalities in early childhood health $\mid$ Review}

\section{RESULTS}

The two components comprising the systematic literature search strategy, which followed the PRISMA guidelines for systematic reviews (10), provided the following results: (i) Medline: 13,270 studies and (ii) CHICOS European child cohort inventory: 4,790 studies (from 77 child cohorts). From this broad search strategy, 616 studies were considered for screening based on title and abstract content, after which 365 were excluded. A full-text article review was performed for the remaining 251 articles. Of these, 201 were considered as eligible to be included in the final review (full reference list is available in the Supplementary Methods S2 online).

\section{Geographical Characteristics}

The 201 identified studies covered 32 countries from all European regions (Table 1). The majority of studies focused on a single country, with only 11 studies presenting results from multiple countries. Two of these studies presented results from 17 European countries. Most studies focused on Western and Northern Europe, with two-thirds of identified studies coming from just six countries: UK (29\% of identified studies), Netherlands (9.5\%), Sweden (9.5\%), Denmark (7.5\%), Germany (6.3\%), and Norway (6\%). Considerably fewer studies were performed in Southern, Central, and Eastern European countries. The majority of the identified studies examined household-level social factors and child health outcomes, while fewer studies focused on neighborhood-level social factors or child development outcomes. Studies that examined associations between neighborhood-level social factors and child development outcomes were performed only in the UK and France.

The general trend across countries was for the reported associations to be significant, with only few country-level differences. A trend of nonsignificant findings was observed only for studies performed in Turkey and Ukraine, where for just 3 out of 10 and 2 out of 11 evaluated social factors, respectively, significant associations were reported. However, it is interesting to note that for these two countries, the results are likely to have been influenced by the choice of outcomes. In both cases, the majority of outcomes were infections, anthropometric outcomes, or digestive disease-outcomes identified by this review to have the weakest associations overall across all studies. Conversely, for other countries, all or almost all associations were reported as significant, such as in Italy (8 out of 8 ), Finland (10 out of 11), and Russia (5 out of 6). In studies performed in the United Kingdom, all 26 evaluated associations between household level social factors and early childhood developmental outcomes were reported as significant.

\section{Socioeconomic Influences on Child Health and Development}

Across the wide range of social factors evaluated, the majority revealed significant associations highlighting the multidimensionality of socioeconomic influences on child health and developmental outcomes (75 and 62\% of evaluated associations for neighborhood-level and household-level social factors, respectively, were reported to be significant) (Table 2)
(Detailed results indicating the direction of the reported association for each specific study are available in the Supplementary Methods S1 online). Overall, 52 different types of outcomes were identified, which were grouped into 13 broad health/ developmental categories spanning the entire spectrum of child health and development (Table 3). Particular health outcomes appeared to be routinely associated with social factors; for example, 9 out of 9 associations evaluating associations between social factors with child general health and illness were reported to be significant. For most health outcomes, the majority of studies demonstrated significant associations, such as for developmental outcomes (43 out of 49 reported associations were significant), asthma (14 out of 15), dental caries (8 out of 9), and psychopathology/mental health problems (6 out of 7). Conversely, the evidence related to other outcomes, such as infection (2 out of 8$)$ and stunting/wasting/poor growth (4 out of 23) and digestive disease (4 out of 17) appeared to be much weaker. However, in the case of stunting/wasting/poor growth, it is interesting to note that all 4 significant, out of 23 evaluated, associations were reported in Commonwealth of Independent States (Kazakhstan, Kyrgyzstan, Russia, and Uzbekistan), indicating region-specific social influences for this key outcome. The summarized findings suggest that socioeconomic factors influence the entire early childhood period, with an increasing trend for significance as children grow up: $57.6 \%$ of evaluated associations were reported to be significant for outcomes measured during the pre- and perinatal period, $54.1 \%$ for infancy, $64 \%$ for toddlerhood, and $68.4 \%$ for the preschool period.

\section{Neighborhood-Level Social Factors}

To define deprivation at the neighborhood level, most studies used composite measures of area-level unemployment, low social class, low income, and lack of car ownership, with $80 \%$ of associations reported as significant. Living in an urban/ high-population density area also tended to show significant associations with poorer health/developmental outcomes, while the evidence regarding surrounding greenness was weak. Only one study focused specifically on the safety of the neighborhood, indicating that living in an unsafe area was associated with increased risk of delay in cognitive development in 3-year olds.

\section{Household-Level Social Factors}

Similar proportions of significant associations were reported for parental social class, income, employment, education (both maternal and paternal), housing tenure (not owning a house), and material deprivation, with around two-thirds of identified associations reported as significant, in the expected direction. For parental employment, a more detailed analysis highlighted differences between studies. Although unemployment (maternal or paternal), living in a workless household, and parental job strain tended to be significantly associated with poorer health and developmental outcomes in children, the opposite pattern was observed for studies which focused on "not being in paid employment" (only one-third of studies 
Table 1. Geographical classification of identified studies

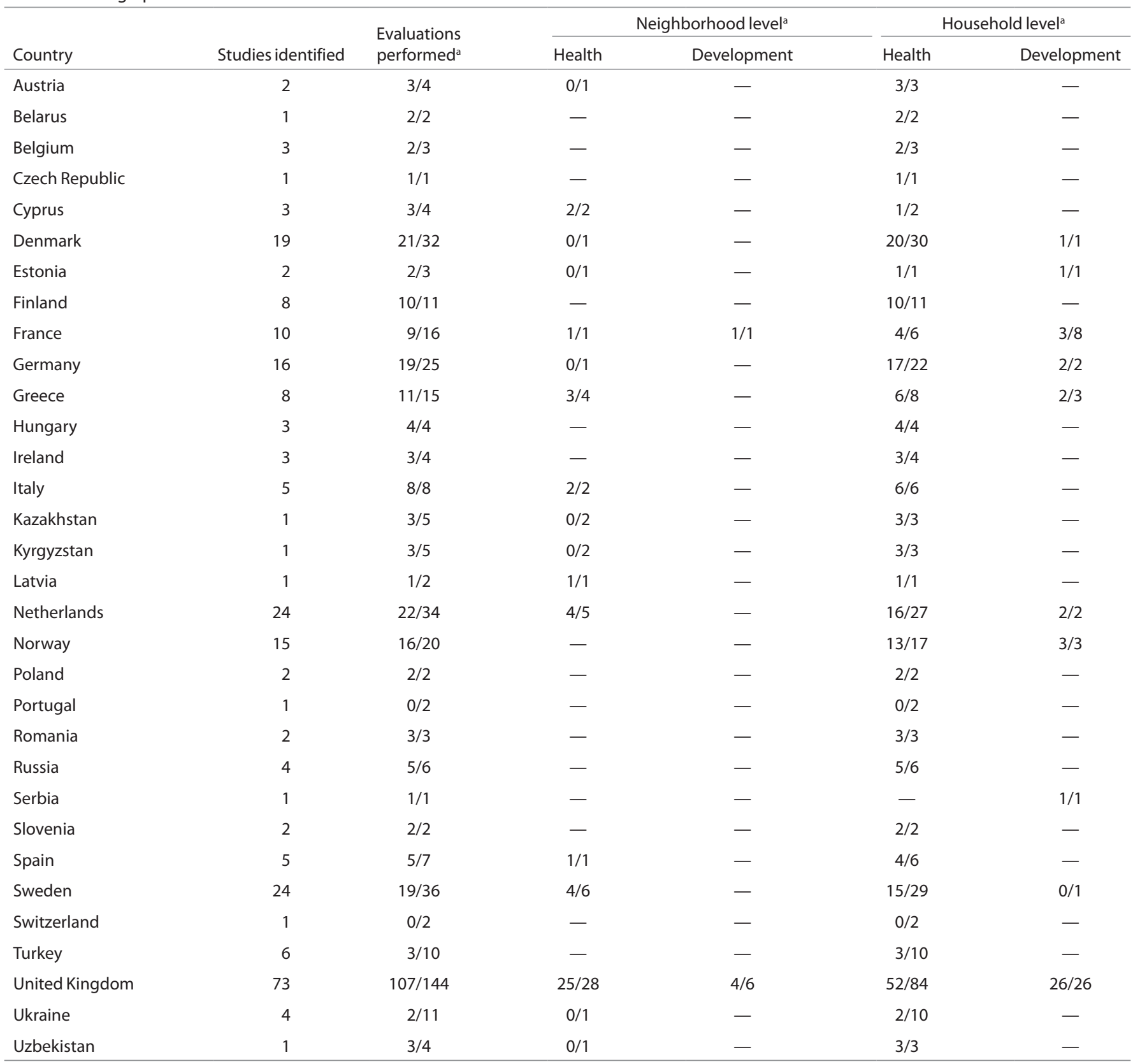

aNumber of significant associations in the expected direction/total number of evaluated associations per country (one unit represents one social factor type evaluated for its association with a child health/developmental outcome. Hence, one unit may refer to one or more evaluations performed within the same social factor category for a specific study).

reported significant associations). The evidence was also weak for household crowding, since the majority of reported associations were nonsignificant.

\section{DISCUSSION}

Overall, the results of the 201 reviewed studies provide strong evidence that neighborhood deprivation, lower parental income/wealth, lower educational attainment, lower occupational social class, parental unemployment, higher parental job strain/heavy physical occupational demands, lack of housing tenure, and material deprivation in the household are all independently associated with a wide range of adverse health and developmental outcomes in early childhood. Insufficient evidence of a consistent association was identified only in relation to household crowding and lack of paid employment in one parent. Social factors appear to influence a wide range of health and developmental outcomes, although important health-specific influences are observed. These findings appear to hold across the majority of European countries, although minor country-level differences were observed. The tendency for evaluations of social influences to report significant associations increased with child age, highlighting potential cumulative effects. Important gaps in the literature were identified, specifically with regard to research evaluating associations 


\section{Inequalities in early childhood health $\mid$ Review}

Table 2. Overview of all evaluated associations ( $n=201$ studies)

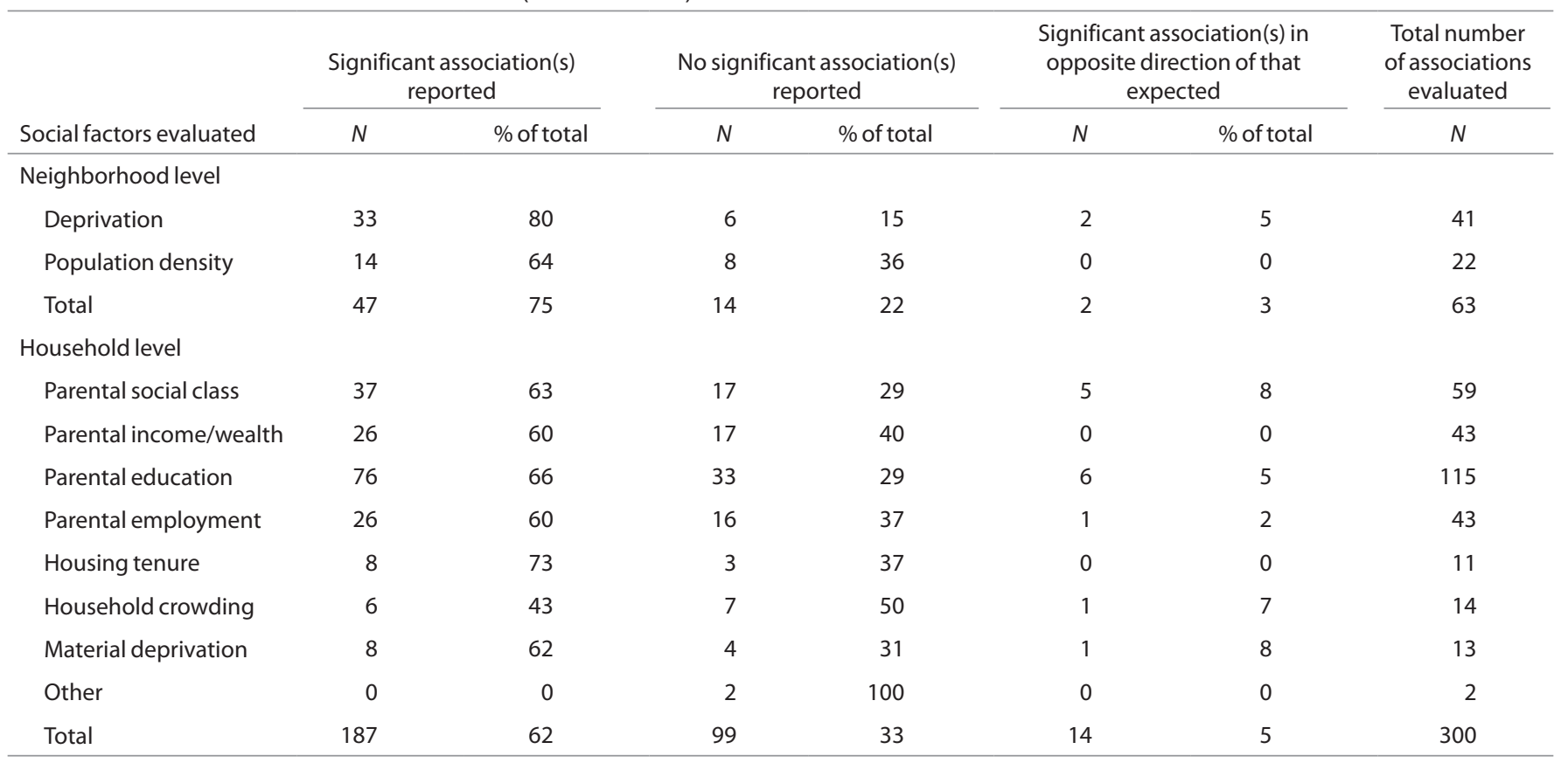

between neighborhood-level social factors and child development outcomes in countries aside the United Kingdom.

\section{Implications for Scientific Knowledge, Policy, and Practice}

The findings of this study carry important implications for scientific knowledge, policy, and practice: (i) that most social factors, at both the neighborhood and household levels, appear to influence early childhood health and development, (ii) that this influence extends across a wide range of adverse health and developmental outcomes in early life, and (iii) that this influence spans the entire continuum of early life.

These findings infer important implications for policy and practice. They indicate that growing up in social disadvantage implies an overall health and developmental disadvantage for children, which, in turn, has been suggested to set individuals on disadvantageous health and development trajectories for the rest of their life course (11). Existing evidence suggests that early onset of a disease/disorder is associated with higher health and financial cost burden. For example, a wide body of research suggests that childhood-onset conduct disorder involves more enduring vulnerabilities that lead to much more chronic and pervasive problems throughout the life course than the adolescent subtype (12). These findings provide further evidence that emphasis should be placed on preventive measures at this early stage of the life course, as it would reduce both the increased subsequent risk and cost (5). Although the potential benefits of early life prevention/intervention have not yet been fully evaluated, a key evidence base is now gradually being established indicating both the clinical and cost effectiveness of such an approach (13).

The finding that multiple social factors, operating at different societal levels (neighborhood and household) entail potential sources of health/developmental disadvantage in early life, highlights the complex embedding, clustering, and cumulative nature of disadvantage. It also indicates the multiplicity of targets that potential interventions could, and should, consider in order to be maximally effective. It provides support to previous work which details how advantage and risk is embedded within families, neighborhoods, and social classes $(14,15)$ and emphasizes the necessity of interventional approaches to be intersectoral, targeting multiple social (and other) factors operating at different levels in order to achieve health equity (6).

\section{Strengths and Limitations of This Review}

This review provides the largest systematic investigation of the association between social factors and adverse childhood health and developmental outcomes and has a number of strengths: (i) inclusion only of contemporary and, therefore, relevant and timely evidence, since only studies published after 2002 with data collected after 1995 were considered, (ii) inclusion only of studies with samples of $\geq 500$ children in their final adjusted analyses, increasing the reliability of findings, (iii) inclusion only of studies published in national or international peer-reviewed journals, (iv) the operational definition of "social factors" to reflect the full spectrum of (potential) socioeconomic disadvantage experienced in early childhood, as well as the different societal levels at which they may operate; similarly, the complete range of health outcomes (including health services utilization) and developmental domains (physical, cognitive, and socioemotional) were included, (v) studies were considered only if they, at least to an extent, adjusted for confounding, hence reducing the possibility that the identified associations are explained by other, nonsocial factors, and (vi) included studies provided evidence from a total of 31 different European countries, covering all European regions (western Europe, Scandinavian, Mediterranean, Balkan states, and central and eastern Europe). 


\section{Review $\mid$ Pillas etal.}

Table 3. Evaluated associations by child health and developmental outcome

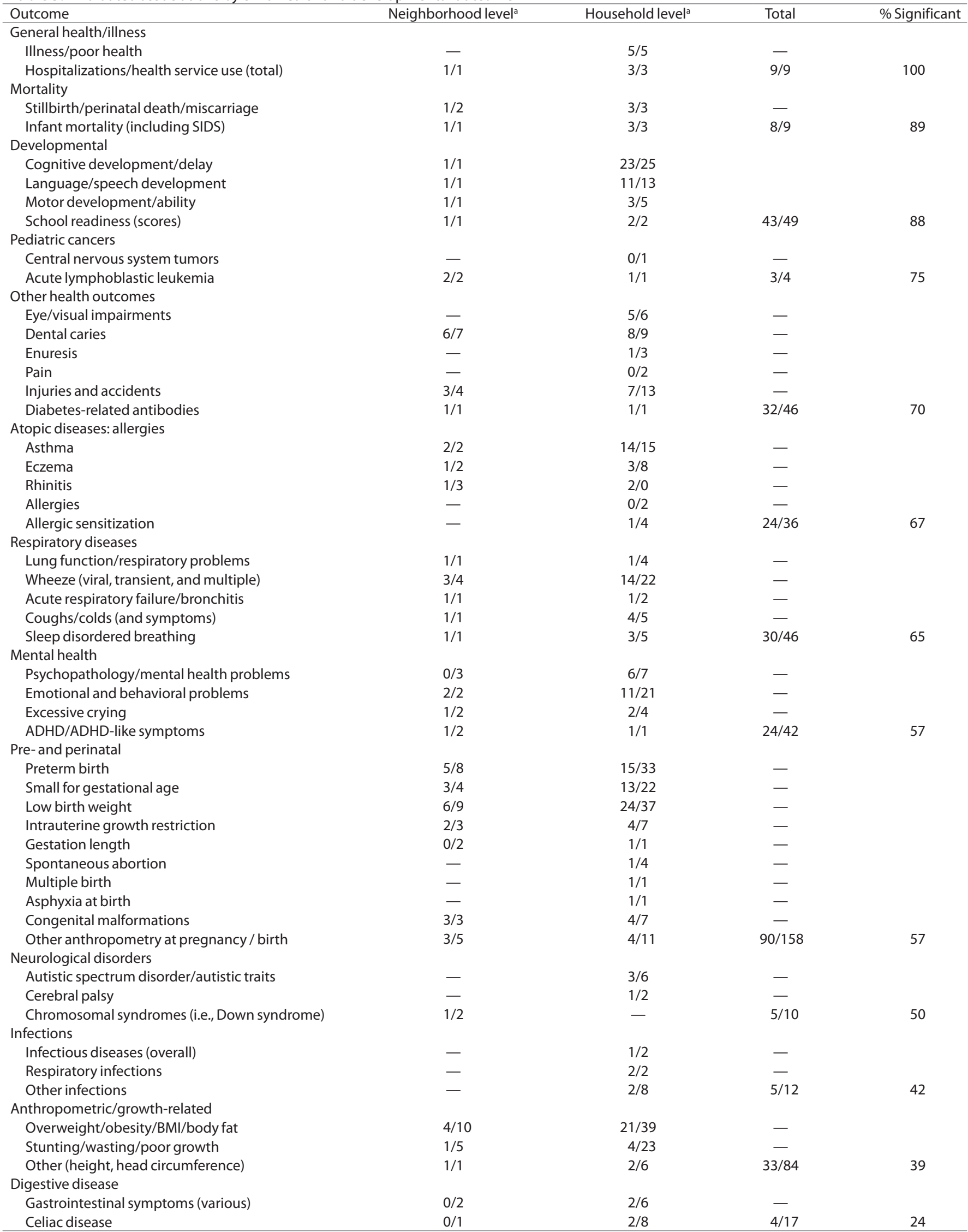

ADHD, attention deficit hyperactivity disorder; SIDS, sudden infant death syndrome

anumber of significant associations in the expected direction/total number of evaluated associations per health/developmental outcome. 


\section{Inequalities in early childhood health $\quad$ Review}

Conversely, this review is also characterized by several limitations: (i) although evidence from a wide range of European countries is provided, this evidence is derived primarily from western and northern European countries ( $70 \%$ of identified studies are from six western and northern European countries, which may be in part due to the English language inclusion criterion), (ii) although only studies which performed some adjustment for potential confounding were considered, many studies may have not performed the full range of potentially necessary adjustments and, therefore, nonsocial confounding of these associations is still possible (e.g., some studies did not adjust for biological factors such as maternal age, maternal BMI, and order of birth that are known to have an important influence on particular early childhood health outcomes), (iii) the proportion of studies reporting significant associations between social factors and child health and development may be an overestimate, due to underreporting of nonsignificant findings (i.e., publication bias) (16).

In the largest systematic review of social inequalities in early childhood health and development to date, a total of 201 studies provided strong evidence that multiple adverse social factors operating at both the household and neighborhood levels are associated with a wide range of adverse health and developmental outcomes throughout the entire period of early childhood. Overall, these findings suggest that the social gradient in health and developmental outcomes observed throughout the remaining life course may be partly explained by gradients initiated in early childhood. These findings suggest multiple opportunities for prevention, early intervention, and intersectoral approaches to tackle the complex embedding, clustering, and cumulative nature of social disadvantage observed in early life.

\section{METHODS}

\section{Search Strategy and Study Inclusion Criteria}

We developed and followed a standard protocol for this review according to the PRISMA guidelines for systematic reviews, which define a rigorous process of study identification, screening, eligibility, and inclusion (10). Studies were considered eligible for inclusion in this review if they were published during the past 10 years (period of publication from 1 July 2002 to 30 June 2012) and used data collected (on average) from 1995 and onwards. This is because the distributions and influences of social factors have been documented to change over time, and this review aimed to reflect the contemporary situation of social inequalities. The WHO European Region classification was used to define the geographical boundaries, which included a total of 53 countries. All included studies were published in English. To ensure study quality, only those published in national or international peer-reviewed journals were considered.

Additionally, studies were considered only if they included quantitative assessments of the associations between social factors in early life and health/developmental outcomes in early childhood. Studies which did not include any adjustment for potential nonsocial confounding and studies with samples of fewer than 500 children in their final adjusted analyses were excluded. In studies with more than one statistical model reported in the results, the model which adjusted for the most factors was considered. Multiple studies based on the same dataset were included only if they performed evaluations of a different social factor, child health/developmental outcome, otherwise only the most recently published study was considered. Studies using datasets with children over $8 \mathrm{y}$ of age were also considered if they included agespecific results within the prenatal to 8 y age range. Across studies, a $5 \%$ significance level was accepted as evidence of statistical significance.
Study identification and data extraction were performed by: (i) searching one scientific literature database (Medline) and (ii) one European child cohort inventory, which included 77 child cohorts from 21 European countries (CHICOS: developing a child cohort research strategy for Europe) (17). Each cohort in the CHICOS inventory was examined through: (i) a search of its online publication list (when available) and (ii) entering the cohort study name as a separate search term in Medline. In addition, relevant references and bibliographies were hand searched for additional studies.

An initial review of titles and abstracts and, subsequently, a full review of all remaining search results, were carried out independently by two reviewers to determine whether they met the criteria for inclusion in this review. All disagreements were resolved via review by a third individual. Where additional information pertaining to a study was necessary, the respective authors were contacted.

\section{Operational Definitions}

Early childhood social factors. Early childhood was defined as "prenatal development to 8 y of age" (1). The term "social factors" was operationally defined to reflect the full spectrum of potential socioeconomic disadvantage experienced in early childhood. For this purpose, the indexes of multiple deprivation were selected as the framework defining the boundaries of what may constitute a "social factor" (18). The indexes of multiple deprivation consist of seven separate "deprivation domains", six of which were considered in this review as the "social factors" in which a child grows up and develops: (i) parental income, (ii) parental employment, (iii) parental education, skills, and training, (iv) access/barriers to housing and services, (v) crime, and (vi) the living environment. Overall, this review considered a specific factor to be a "social factor" if it could be viewed in a socioeconomic context (i.e., bed sharing was considered a social factor if it was reported in the context of household crowding/deprivation, but not if the infant shared a bed with parents due to young age). The seventh index of multiple deprivation domain ("health deprivation and disability") was excluded, since this was not a social factor per se and overlapped with our outcomes relating to child health. Factors relating to ethnicity, parental behavior, and environmental pollution were not considered in this review.

Childhood health and developmental outcomes. To measure the impact of social factors on early childhood health, a comprehensive range of possible health measures/outcomes was considered. Early childhood development encompasses a wide range of developmental outcomes, covering all aspects of physical, cognitive, and socioemotional development. Childhood health behaviors such as physical activity and sedentary behavior were not considered in this review.

\section{SUPPLEMENTARY MATERIAL}

Supplementary material is linked to the online version of the paper at http:// www.nature.com/pr

\section{ACKNOWLEDGMENTS}

The authors are grateful for constructive comments on an earlier version of the manuscript given by the following members of the DRIVERS consortium: Hanno Hoven and Johannes Siegrist, University of Duesseldorf; Olle Lundberg, Stockholm University; and Claudia Marinetti and Linden Farrer, EuroHealthNet, Brussels.

\section{STATEMENT OF FINANCIAL SUPPORT}

All phases of this study were supported by a European Union FP7 grant, DRIVERS, EC-GA HEALTH-F3-2011-278350.

\section{REFERENCES}

1. World Health Organization Early Child Development Knowledge Network (WHO ECDKN). Early Child Development: A Powerful Equalizer. Final Report of the Early Childhood Development Knowledge Network of the Commission on Social Determinants of Health. Geneva: World Health Organization, 2007.

2. Shonkoff J, Phillips D, eds. From Neurons to Neighbourhoods: The Science of Early Childhood Development. Washington, DC: National Academy Press, 2000. 
3. Gale CR, O'Callaghan FJ, Bredow M, Martyn CN; Avon Longitudinal Study of Parents and Children Study Team. The influence of head growth in fetal life, infancy, and childhood on intelligence at the ages of 4 and 8 years. Pediatrics 2006;118:1486-92.

4. Knudsen EI, Heckman JJ, Cameron JL, Shonkoff JP. Economic, neurobiological, and behavioral perspectives on building America's future workforce. Proc Natl Acad Sci USA 2006;103:10155-62.

5. Doyle O, Harmon CP, Heckman JJ, Tremblay RE. Investing in early human development: timing and economic efficiency. Econ Hum Biol 2009;7:1-6.

6. Commission on Social Determinants of Health. Closing the Gap in a Generation. Geneva: World Health Organization, 2008.

7. WHO Regional Office for Europe. Review of Social Determinants and the Health Divide in the WHO European Region: Final Report. Copenhagen, Denmark: WHO Regional Office for Europe, 2013.

8. Bronfenbrenner U. Ecological systems theory. In: Vasta R, ed. Annals of Child Development: Six Theories of Child Development-Revised Formulations and Current Issues. Boston, MA: JAI Press, 1989:187-249.

9. Sameroff A, Fiese B. Transactional regulation and early intervention. In: Meisels SJ, Shonkoff J, eds. Early Intervention: A Handbook of Theory, Practice and Analysis. New York: Cambridge University Press, 1989.

10. Moher D, Liberati A, Tetzlaff J, Altman DG; PRISMA Group. Preferred reporting items for systematic reviews and meta-analyses: the PRISMA statement. PLoS Med 2009;6:e1000097.
11. Kuh D, Ben-Shlomo Y, Lynch J, Hallqvist J, Power C. Life course epidemiology. J Epidemiol Community Health 2003;57:778-83.

12. Brown SA, Gleghorn A, Schuckit MA, Myers MG, Mott MA. Conduct disorder among adolescent alcohol and drug abusers. J Stud Alcohol 1996;57:314-24.

13. Pillas D, Naicker K, Colman I, Hertzman C. Public health, policy, and practice: implications of life course approaches to mental illness. In: Koenen K, Rudenstine S, Susser E, Galea S, eds. A Life Course Approach to Mental Disorders. Oxford: Oxford University Press, 2013.

14. Elder GH, Shanahan MJ. The life course and human development. In: Lerner R, Damon W, eds. The Handbook of Child Psychology. 6th edn. New York: Wiley, 2007:665-715.

15. Wolff J, de-Shalit A. Disadvantage. Oxford: Oxford University Press, 2007:119-32.

16. Dwan K, Altman DG, Arnaiz JA, et al. Systematic review of the empirical evidence of study publication bias and outcome reporting bias. PLoS One 2008;3:e3081.

17. CHICOS. Developing a Child Cohort Research Strategy for Europe, 2013. (http://www.chicosproject.eu.)

18. UK Department of Communities and Local Government. English Indices of Deprivation 2010. (https://www.gov.uk/government/publications/ english-indices-of-deprivation-2010.) 